\title{
Probabilistic Evaluation Method of Interconnectable Capacity for Wind Power Generation Using Actual Data
}

\author{
T. Yoshida ${ }^{1}$, M. Kato ${ }^{1}$ and K. Kashima ${ }^{2}$ \\ ${ }^{1}$ Department of Electrical and Electronic Engineering, \\ Tokyo Denki University, 5, Senju-Asahi-cho, Adachi-ku, Tokyo, Japan, 120-8551 (Japan) \\ ${ }^{2}$ Kyoto University, Yoshida-honmachi, Sakyo-ku, Kyoto, Japan, 606-8501 (Japan)
}

\begin{abstract}
New analysis method of power quality using stochastic linearization is proposed to evaluate stochastically interconnectable capacity for wind power generation. This is based on mathematical model of load frequency control systems, which involve nonlinear and dynamic characteristics. Nonlinear generator model is linearized to analyse power quality through stochastic linearization. However, accuracy evaluation of model due to linearization has not been examined using actual data. In this paper, we stochastically evaluate accuracy of linearized model and interconnectable capacity for wind power generation.
\end{abstract}

\section{Key words}

Wind power generation, Interconnectable capacity, Probabilistic method, Stochastic linearization

\section{Introduction}

Recently, introduction of renewable energy (RE) is being promoted all over the world due to prevention of global warming and improvement of energy security. RE is power generation using a variety of natural energy such as wind power (WP) and photovoltaic (PV), which is expected as new energy supply. In Europe, they have been introduced aggressively centering on Spain and Germany [1]. In Japan, Act on Special Measures Concerning Procurement of Electricity from Renewable energy Sources by Electricity Utilities has been enacted to promote introduction of RE. At the same time, Feed-in tariff (FIT) targeted at WP and PV and so on has been implemented. In the near future, introduction amount of WP and PV is expected to additionally increase. However, WP and PV outputs are fluctuated by weather conditions. When they are heavily introduced to power systems, keeping balance of demand and supply might be difficult, which affects power quality significantly.

Currently, Japanese power companies except for major ones, are limiting maximum capacity of interconnectable WP as shown in Table 1 [2]. This comes from feature of Japanese power system. Japanese power system, different from meshed power system like Europe, is a longitudinal transmission system. This comes from landform of Japan. In addition, power system frequency is different in eastern $(50 \mathrm{~Hz})$ and western part $(60 \mathrm{~Hz})$, which are interconnected via frequency converter stations of three locations. Each
Table 1. Interconnectable capacity of WP (As of the end of March, 2014)

\begin{tabular}{c||c}
\hline Electric Power Campany & Max Capacity [MW] \\
\hline \hline Hokkaido E.P.C & 560 \\
\hline Tohoku E.P.C & 2000 \\
\hline Tokyo E.P.C & - \\
\hline Chubu E.P.C & - \\
\hline Kansai E.P.C & - \\
\hline Hokuriku E.P.C & 450 \\
\hline Chugoku E.P.C & 1000 \\
\hline Shikoku E.P.C & 600 \\
\hline Kyusyu E.P.C & 1000 \\
\hline Okinawa E.P.C & 250 \\
\hline
\end{tabular}

power system is basically interconnected only one transmission route with two lines. Imbalance of demand and supply in each power system brings about large fluctuation of tie line power flow, which would disturb economic system operation. Therefore, keeping balance of demand and supply within each power system is essential for economic system operation.

WP output is likely to fluctuate greatly even at low demand of midnight. Demand and supply adjustment is conducted by available thermal power unit. If it reaches its minimum output limit, surplus power due to lack of adjusting power comes about. Accordingly, the power system frequency decays. Therefore, Japanese power systems limit interconnectable capacity of WP for frequency not to deviate from the allowable range. Maximum interconnectable capacity is based on calculation results assumed worse cases in system operation. However, FIT requires disconnection of RE generation from power system if power quality drastically gets worse. It is reported that the probability that WP output fluctuation is maximum is extremely low due to analysis of many actual data [3]. Therefore, present interconnectable capacity of WP is likely to be underestimated. This paper proposes advanced evaluation method of interconnectable capacity for WP using probabilistic approach. 
In the previous work [4], authors proposed probabilistic evaluation method of interconnectable capacity for WP, which is considered nonlinear and dynamic characteristics in power systems. The idea of proposed method is based on the fact that if white noise is inputted in linear model we can easily estimate stationary probability distribution of output. First, applied is WP output model with white noise and first-order lag element, which is based on distribution of frequency domain obtained by Fourier analysis of actual data of WP output and follows a normal distribution. Second, employed is linearization of nonlinear generator model with saturation and rate limiter. Using these methods, it has been shown that the frequency deviation distribution is calculated without numerical simulations. Additionally, adequacy of WP output model has been verified there.

Accuracy evaluation of the model due to linearization, however, has not been conducted yet. In this paper, we stochastically evaluate accuracy of model due to linearization and interconnectable capacity for wind power generation.

\section{Simulation Method}

Evaluated stochastically is accuracy of the linearized model and interconnectable capacity for WP by frequency control simulation using Monte Carlo method. When a large amount of WP is introduced in the power system, adjusting power for load frequency control (LFC) with fluctuation period shorter than 20 minutes may become insufficient. Therefore, the simulation is focussed on fluctuation period shorter than 20 minutes.

To run frequency control simulations using Monte Carlo method, 10,000 cases of actual data for WP output and demand fluctuation are inputted to the system model explained later. Used data are randomly and independently extracted for an hour data and combined with WP output and demand fluctuation. Thus, not only for the worst case in the conventional method, but also for every demand and WP condition the simulation is run. Using frequency deviation obtained by the simulation, probability distribution is calculated. Definitely, interconnectable capacity for WP that satisfies frequency management target value $( \pm 0.2 \mathrm{~Hz})$ of the power system frequency is estimated.

\section{Simulation Conditions}

\subsection{Simulation Model}

A. System Model

Japanese power companies have operated power system based on the idea that imbalance of demand and supply is adjusted by each power system. Additionally, interconnectable capacity for current WP is decided assuming no compensation by other interconnected power system. Therefore, system model is defined as a single power system as shown in Fig. 1.

Fig. 1 is represented by mathematical model, which is widely used for the evaluation of demand and supply adjustment. The LFC applied here is Flat Frequency Control (FFC). First, WP output and demand fluctuation is inputted to the system model as system disturbance. WP model uses actual data, which is not considered detailed

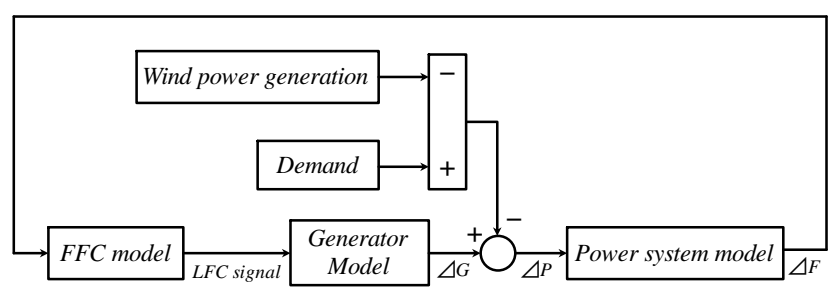

Fig. 1 System model

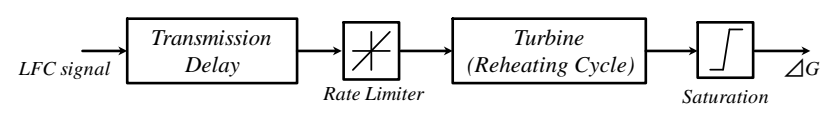

Fig. 2 Generator model (Nonlinear model)

Table 2. Saturation and rate limiter depending on the system capacity

\begin{tabular}{c|c||c||c}
\hline \multicolumn{2}{c||}{ System Capacity [MW] } & Saturation [MW] & Rate Limiter [MW/min] \\
\hline \hline Max & 4515 & \pm 45.2 & \pm 22.6 \\
\hline Min & 3343 & \pm 33.4 & \pm 16.7 \\
\hline
\end{tabular}

modelling of wind power generator. Similarly, demand model also uses actual data. Difference of those synthetic fluctuation and output of generator model is calculated as imbalance of demand and supply $\Delta P$. Frequency deviation $\Delta F$ is calculated by

$$
\Delta F=\frac{\Delta P}{K}
$$

where system constant $K$ is $10 \% \mathrm{MW} / \mathrm{Hz}$ for system capacity. (1) is applied by ignoring a transient phenomenon shorter than a few seconds. However, as the power system has the transient phenomenon of a few seconds, the power system model includes first-order lag element as

$$
P_{1}(s)=\frac{1}{3 s+1} .
$$

$\Delta F$ is converted into LFC signal to compensate the $\Delta P$ in FFC model. Output of the generator model flexibly changes depending on the LFC signal. Control period of LFC is defined as 10 s.

\section{B. Generator Model \\ 1) Nonlinear Model}

As shown in Fig. 2, generator model is only taken into account turbine model and rate limiter, saturation, time delay component due to transmission delay of LFC signal. Additionally, the model in Fig. 2 is nonlinear system to have limiter characteristics of saturation and rate limiter, transmission delay. Electric Power System Council of Japan (ESCJ) is defined LFC adjustment capability for fluctuation period shorter than 20 minutes as $2 \%$ of the system capacity [5]. Therefore, the saturation is assumed to be $\pm 1 \%$ of the system capacity. The rate limiter is assumed to be $\pm 5 \% \mathrm{MW} / \mathrm{min}$ of thermal power unit capacity. Here, thermal power unit capacity is assumed to be $10 \%$ of the system capacity. The transmission delay is assumed to be 10s. Turbine model is modelled by firstorder lag element as

$$
P_{2}(s)=\frac{1}{1 / 0.15 \cdot s+1}
$$

Because after-mentioned system capacity is in the range of $3343 \mathrm{MW}$ from $4515 \mathrm{MW}$, the saturation and the rate 


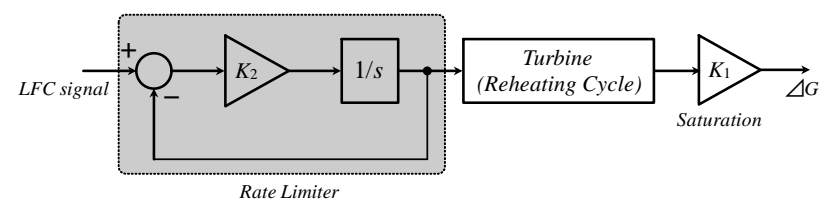

Fig. 3 Generator model (Linearized model)

Table 3. Result of linearized gains

\begin{tabular}{c||c|c||c|c}
\hline \multicolumn{1}{c||}{$\begin{array}{c}\text { Interconnectable } \\
\text { Capacity of WP [MW] }\end{array}$} & \multicolumn{2}{c||}{ Saturation $K_{1}$} & \multicolumn{2}{c}{ Rate Limitter $K_{2}$} \\
\cline { 3 - 5 } & Min & Max & Min & Max \\
\hline \hline 500 & 0.9955 & 0.9999 & 0.1751 & 0.3909 \\
\hline 600 & 0.9814 & 0.9987 & 0.1153 & 0.2358 \\
\hline 700 & 0.9534 & 0.9939 & 0.0829 & 0.1607 \\
\hline 800 & 0.9120 & 0.9830 & 0.0627 & 0.1186 \\
\hline 900 & 0.8605 & 0.9645 & 0.0490 & 0.0920 \\
\hline 1000 & 0.8033 & 0.9382 & 0.0391 & 0.0737 \\
\hline 1100 & 0.7447 & 0.9051 & 0.0318 & 0.0604 \\
\hline 1200 & 0.6881 & 0.8668 & 0.0262 & 0.0503 \\
\hline 1300 & 0.6354 & 0.8251 & 0.0220 & 0.0425 \\
\hline 1400 & 0.5877 & 0.7818 & 0.0186 & 0.0362 \\
\hline 1500 & 0.5449 & 0.7386 & 0.0159 & 0.0311 \\
\hline
\end{tabular}

limiter in each case of the simulation are in the range of maximum from minimum as shown in Table 2 .

\section{2) Linear Model}

Based on the previous work [4], the saturation and the rate limiter of nonlinear elements in Fig. 2 are linearized. Its linearization is calculated on equal terms with the nonlinear model. As shown in Fig. 3, linearized model expresses the saturation in linearized gain $K_{1}$ and the rate limiter in feedback system of linearized gain $K_{2}$ and integrator. Time delay component due to transmission delay of LFC signal is ignored in Fig. 3 due to very small impact by preliminary study. The turbine model, as with nonlinear model, is modelled by the first-order lag element as (3).

Since the system capacity is in the range of $3343 \mathrm{MW}$ from $4515 \mathrm{MW}$, linearized gain $K_{1}$ and $K_{2}$ in each case of the simulation are in the range of maximum from minimum as shown in Table 3 based on the method stated in the previous work [4].

\subsection{WP Output Data}

WP output uses actual data in May. Fluctuation period shorter than 20 minutes is used, which is equivalent to control period of LFC. When WP is widely introduced, it is demonstrated that the fluctuation of total WP output is smoothed [3]. This smoothing effect of WP is assumed as $20 \%$ against maximum WP capacity during 20 minutes [5]. As mentioned before, WP output data inputted to WP model in Fig. 1 is randomly extracted 10,000 cases. Each case is the data of 3600 s length. For each case, WP output is represented as deviation from the initial value because balance of demand and supply is equilibrium at the simulation start.

\subsection{Demand Fluctuation Data}

Demand fluctuation data uses actual data in May. Fluctuation period shorter than 20 minutes is used as well as WP output. Demand fluctuation data inputted to demand model in Fig. 1 is randomly extracted 10,000 cases as well. Each case is the data of 3600s length. For each case, demand fluctuation is deviation from initial value because balance of demand and supply is equilibrium at the simulation start.

\subsection{System Capacity}

System capacity is actual data in May. It is hourly maximum demand, which is in the range of 3343MW from $4515 \mathrm{MW}$. Because demand fluctuation data is randomly extracted 10,000 cases, system capacity of each case is decided by demand profile of extracted time zone. Therefore, the saturation and the rate limiter in the generator model are calculated depending on the system capacity of each case.

\subsection{Evaluation Method}

To evaluate interconnectable capacity for WP, standard deviation of frequency deviation $\sigma$ is used, which is assumed that frequency distribution in the power system can approximate by the normal distribution. Frequency management target value of Japanese power companies is generally $\pm 0.2 \mathrm{~Hz}$ of the power system frequency. The power system frequency, except for large failures does not significantly fluctuate. Therefore, $3 \sigma$ value is used. When $3 \sigma$ value equals $0.2 \mathrm{~Hz}$, WP is interconnectable with cumulative probability of $99.7 \%$.

\section{Simulation Result}

Here, interconnectable capacity for WP is evaluated based on the evaluation method. As shown in Table 4 and 5, interconnectable capacity for WP is estimated as $1200 \mathrm{MW}$ applying the nonlinear model and 1300MW applying the linear model. Standard deviation of the nonlinear model is greater than the linear model. Therefore, result of the simulation applying the nonlinear model is rather safe for system operation. Although this result has satisfied the frequency management condition, the frequency deviation may significantly deviate from frequency management target value for the worst case. This may be addressed by disconnection of WP from the power system or other measures.

As shown in Fig. 4, the standard deviation of the linear model has an error compared with the nonlinear model. This stems from the error that occurred by linearizing nonlinear elements of generator model. As shown from Fig. 5 to Fig. 9, compared with both models, shape of frequency deviation distribution is extremely similar. However, compared with normal distribution that has same variance with the simulation result, the simulation result concentrates on near the average. Here, WP output distribution that is used in this paper is a little bit different from the normal distribution as shown in Fig. 10.

When the variance of the input random variable is small enough compared to the threshold of the saturation, the effect of the saturation is small, and consequently the linearized gain is close to 1 . Conversely, the variance is large enough, the input/output ratio is close to 0 with a high probability, and thus the linearized gain is close to 0 . In other cases, the linearized gain takes the value in between that minimizes the approximation error variance. 
Table 4. Result of interconnectable capacity for WP in the nonlinear model

\begin{tabular}{c||c||c||c||c}
\hline $\begin{array}{c}\text { Interconnectable } \\
\text { Capacity of WP [MW] }\end{array}$ & Average [Hz] & $\begin{array}{c}\text { Standard } \\
\text { Deviation [Hz] }\end{array}$ & Max [Hz] & Min [Hz] \\
\hline \hline 500 & -0.000278 & 0.0438 & 0.289 & -0.262 \\
\hline 600 & -0.000266 & 0.0462 & 0.313 & -0.284 \\
\hline 700 & -0.000257 & 0.0490 & 0.327 & -0.306 \\
\hline 800 & -0.000252 & 0.0520 & 0.360 & -0.336 \\
\hline 900 & -0.000245 & 0.0553 & 0.389 & -0.377 \\
\hline 1000 & -0.000239 & 0.0588 & 0.427 & -0.417 \\
\hline 1100 & -0.000235 & 0.0624 & 0.461 & -0.459 \\
\hline 1200 & -0.000228 & 0.0662 & 0.489 & -0.511 \\
\hline 1300 & -0.000219 & 0.0702 & 0.519 & -0.561 \\
\hline 1400 & -0.000203 & 0.0743 & 0.553 & -0.604 \\
\hline 1500 & -0.000183 & 0.0785 & 0.578 & -0.641 \\
\hline
\end{tabular}

Table 5. Result of interconnectable capacity for WP in the linear model

\begin{tabular}{c||c||c||c||c}
\hline $\begin{array}{c}\text { Interconnectable } \\
\text { Capacity of WP [MW] }\end{array}$ & Average [Hz] & $\begin{array}{c}\text { Standard } \\
\text { Deviation [Hz] }\end{array}$ & Max [Hz] & Min [Hz] \\
\hline \hline 500 & -0.000440 & 0.0389 & 0.262 & -0.254 \\
\hline 600 & -0.000444 & 0.0416 & 0.277 & -0.269 \\
\hline 700 & -0.000450 & 0.0445 & 0.290 & -0.301 \\
\hline 800 & -0.000460 & 0.0476 & 0.317 & -0.330 \\
\hline 900 & -0.000472 & 0.0509 & 0.345 & -0.356 \\
\hline 1000 & -0.000488 & 0.0544 & 0.372 & -0.378 \\
\hline 1100 & -0.000505 & 0.0582 & 0.397 & -0.401 \\
\hline 1200 & -0.000523 & 0.0622 & 0.420 & -0.438 \\
\hline 1300 & -0.000541 & 0.0644 & 0.445 & -0.479 \\
\hline 1400 & -0.000560 & 0.0709 & 0.470 & -0.523 \\
\hline 1500 & -0.000578 & 0.0755 & 0.500 & -0.568 \\
\hline
\end{tabular}

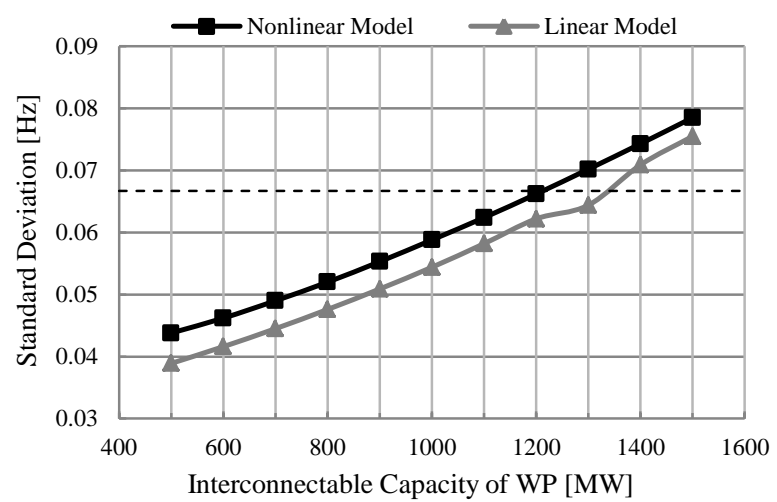

Fig. 4 Standard deviation of frequency deviation

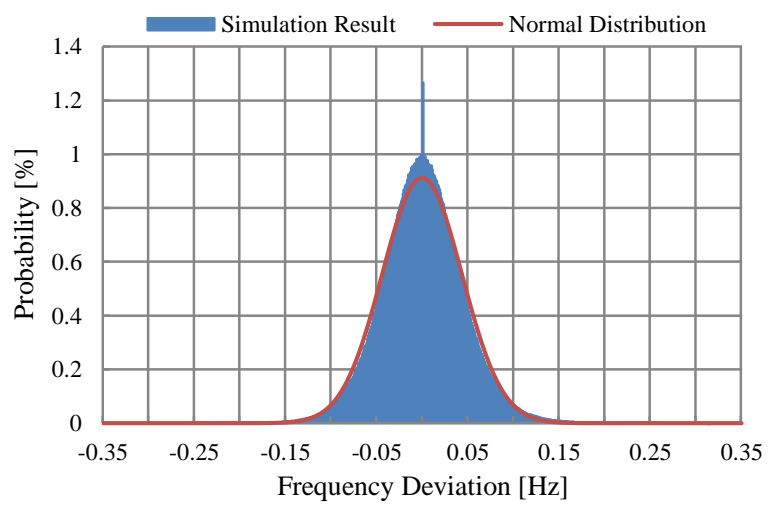

(a) Nonlinear model

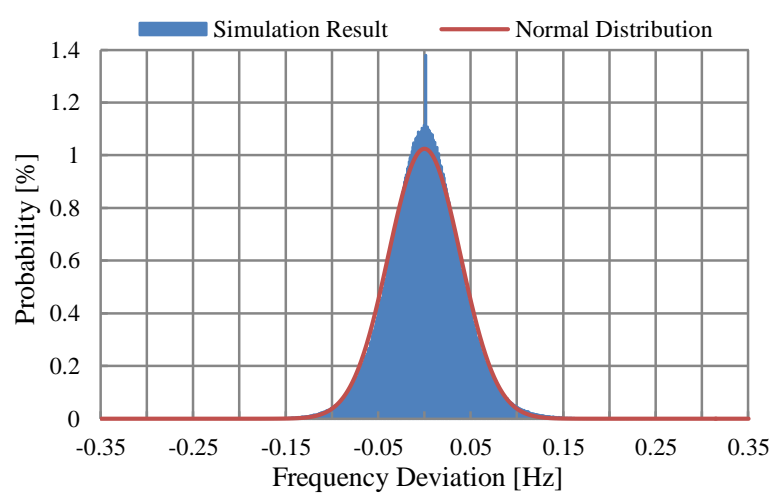

(b) Linear model

Fig. 5 Frequency deviation distribuntion (Interconnectable capacity of WP is $500 \mathrm{MW}$ )

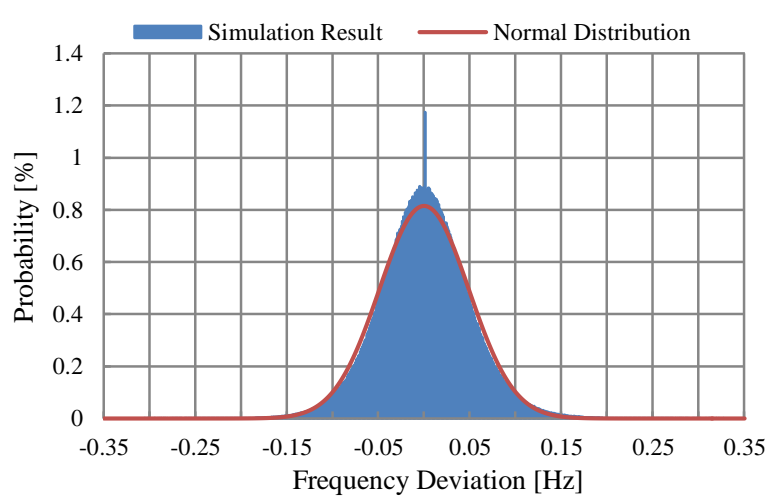

(a) Nonlinear model

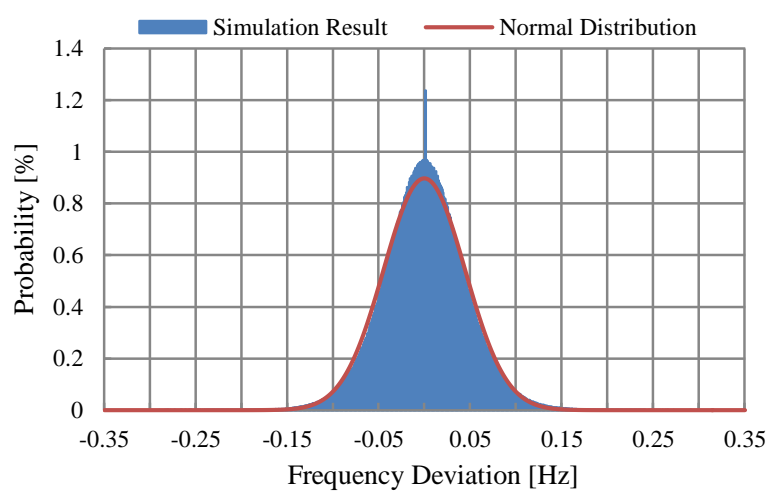

(b) Linear model

Fig. 6 Frequency deviation distribuntion (Interconnectable capacity of WP is $700 \mathrm{MW}$ )

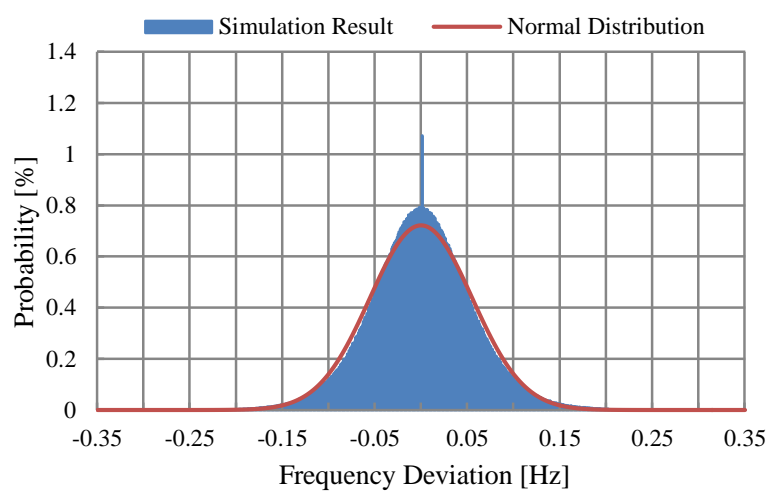

(a) Nonlinear model 


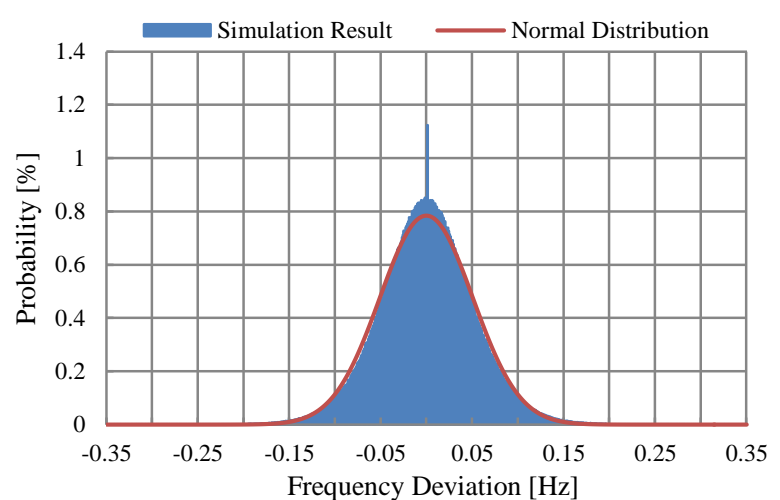

(b) Linear model

Fig. 7 Frequency deviation distribuntion (Interconnectable capacity of WP is $900 \mathrm{MW}$ )

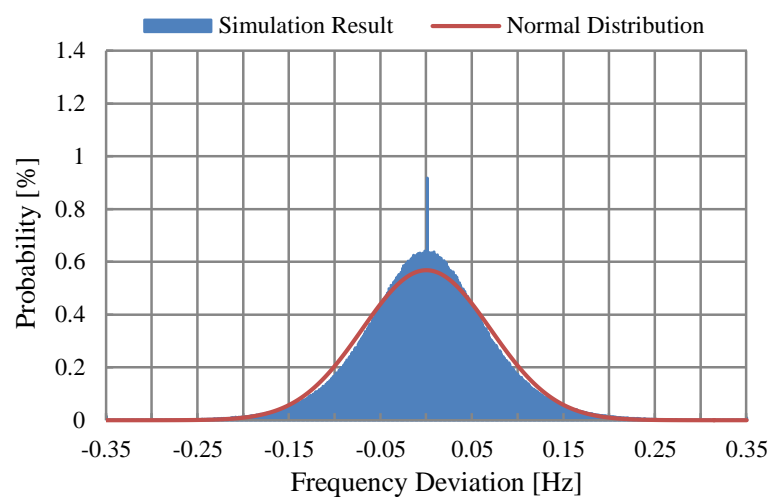

(a) Nonlinear model

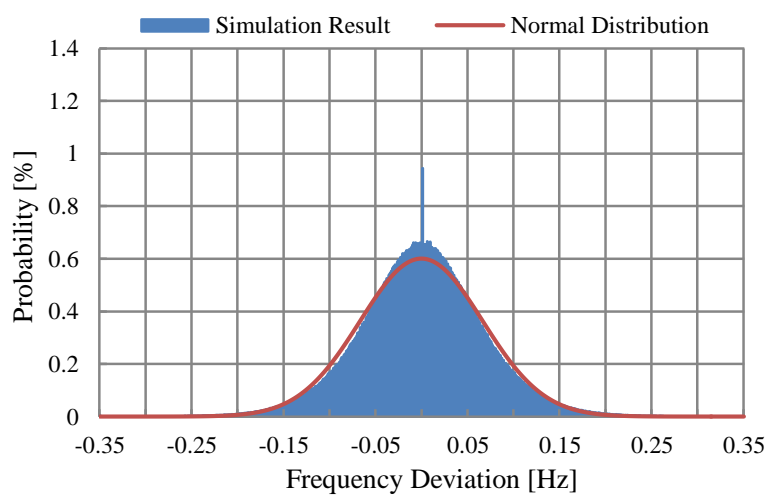

(b) Linear model

Fig. 8 Frequency deviation distribuntion (Interconnectable capacity of WP is $1300 \mathrm{MW}$ )

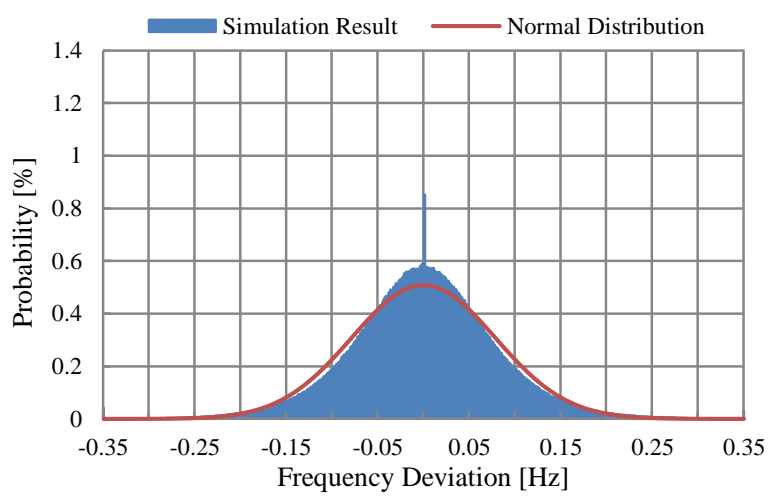

(a) Nonlinear model

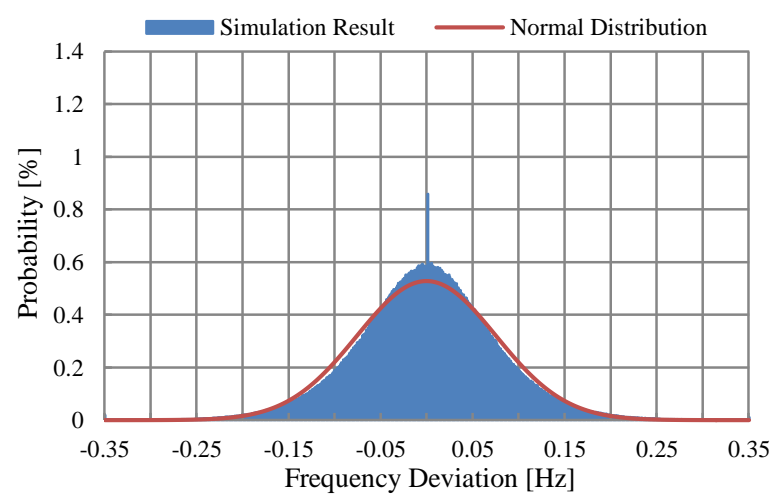

(b) Linear model

Fig. 9 Frequency deviation distribuntion (Interconnectable capacity of WP is $1500 \mathrm{MW}$ )

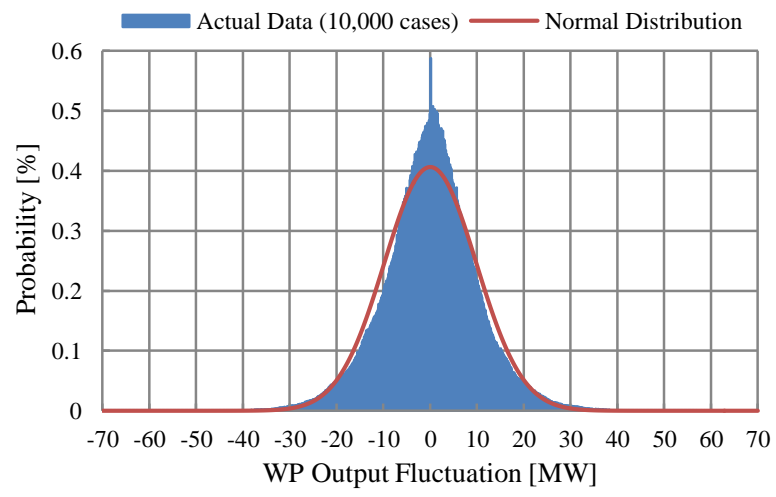

Fig. 10 Example of WP output distribution (Interconnectable capacity of WP is $500 \mathrm{MW}$ )

\section{Conclusion}

In this paper, we have stochastically evaluated accuracy of the linearized model and interconnectable capacity for WP. In the future, we are going to analyse critical element on the expansion of interconnectable capacity of WP. Considering the properties of the linearized gain described above, we expect that the effect of the saturation can be evaluated by using the linearized gain, and thus it affects interconnectable capacity for WP dominantly. In addition, we are going to examine including fluctuation period longer than 20 minutes of WP output and demand fluctuation.

\section{References}

[1] "Powering Europe : Wind energy and the electricity grid" European Wind Energy Association, 2010

[2] http://www.fepc.or.jp/environment/new_energy/renkei/sw_in dex_01/ (in Japanese)

[3] http://jwpa.jp/2011_pdf/89-04news.pdf (in Japanese) Japan Wind Energy Association

[4] K. Kashima, M. Kato, J.-I. Imura, K. Aihara, Eur. Phys. J. Special Topics, EDP Sciences, Springer-Verlag 2014

[5] http://www.escj.or.jp/energy/wg/pdf/report_windpower_wg. pdf (in Japanese)

[6] K. Kashima, M. Kato, J.-I. Imura, K. Aihara, Proc. Annual Conf. Power and Energy Society, IEE Japan 155 (2013) (in Japanese) 